\title{
A CONCEPT OF GENERAL MEANING: SELECTED THEORIES IN COMPARISON TO SELECTED SEMANTIC AND PRAGMATIC THEORIES
}

\author{
ROMAN KALISZ \\ University of Gdańsk \\ kalrom@wp.pl
}

\begin{abstract}
The paper discusses a concept of general meaning with reference to various relevant semantic and pragmatic theories. It includes references to Slavic axiological semantics (e.g. Krzeszowski (1997); Puzynina (1992)), Wierzbicka's (e.g. 1980, 1987) atomic expressions and classical pragmatics theories, such as speech acts, Gricean theory of conversational implicature, politeness theory and and relevance theory.
\end{abstract}

Keywords: general meaning, semantics, pragmatics

\section{The rudiments of the concept of general meaning}

In this section I would like to introduce a concept of general meaning which I claim is a valid notion in comparing cultural and linguistic phenomena across various language communities. I claim that at this level of semantic investigations similarities and differences among various cultural phenomena are most salient.

Let us start with exposition of the concept of general meaning which I have been developing for the last two years.

The concept of general meaning is connected with degree of schematicity of semantic description and perception. I claim here that it is possible to distinguish a level for basic values in a given culture. Let us present the following example which is an extract of an interview of a journalist with Muhammad Ali:

$$
\begin{aligned}
& \text { A. A journalist: You are extremely truculent! } \\
& \text { B. Ali: If it's good! I'm that! }
\end{aligned}
$$

Ali was not interested in the detailed meaning of the word truculent but was interested in what we call here general meaning. Here the aspect of general meaning is connected with evaluation of a situation, more precisely, with description of Ali in terms of 'good' or 'bad'. I $\mathrm{n}$ fact the term truculent may in itself be rather neutral idiosyncratic for its interpretation in the above terms. However, this aspect of understanding of the word truculent interested Muhammad Ali most. I claim that understanding and producing convictions in terms of a scale ranging from 'good' or 'positive' evaluation of an event 
or a situation, through less enthusiastic evaluation in terms of positive indication, through neutral stance toward judgment in weaker and later stronger negative terms. The positive-negative or good-bad scale is one of the most fundamental parameters in human cognition through which humans assess observations of the world. Krzeszowski (1997) claims that every linguistic expression is charged axiologically. In other words every expression is confronted with good-bad parameter. The remaining two parameters are truth-falsehood spectrum and beautiful-ugly continuum. The location of a given expression on one or more continua presents general meaning of this expression. The general meaning is, then instantiated as a detailed meaning of the expression.

The utterance: This soup is not good represents prototypically a general meaning connected with location of the expression on the scale close to bad pole of the spectrum, whereas particular instantiations which contain obviously their general meaning too, may be as follows:
a. This soup is not tasty.
b. This soup is contaminated.
c. This soup is too salty.
d. The consumption date for this soup is expired.

I propose three spectra which accommodate general meaning. An expression can be related to more than one spectrum:

Peter claims that Mary likes impressionists.

In the above utterance Peter is convinced that what he maintains (proposition) is true. The proposition contains the verb like which signals positive attitude toward the object of the utterance which is connected with art. Therefore, the scale beautiful-ugly enters the picture.

After some examinations connected with questionnaires too, these scales seem to be the most significant for human perception in Western culture. Obviously, some other scales are possible such as strong-weak, wide-narrow, high-low etc. However, they seem to be secondary and reducible to the three major scales. In some contexts other scales may be in focus and may be more important than others. In formula 1 stable fast-slow scale is probably the most important of all scales and all other are subordinate to this one. In other than Western cultures some other scales than our three presented may be more salient. Nevertheless, the cultural differences would be related to different assessment of various phenomena on various scales. To be consistent with the culinary subject, pork is not i.e. acceptable in Jewish culture, beef is not acceptable in Hinduism although for different reasons than pork in Jewish culture. At this level, major cultural differences can be noted stemming from different views of the world. Liberalism is viewed as bad socialist, let alone communist tradition. However, it is highly praised in other circles e.g. those represented in the journal Najwyższy Czas (The High Time). What is true? What is false? What is wrong? What is right? What is good? What is bad? 
What is nice? What is ugly? Are questions often differently answered by many cultures, circles, political parties, unions, religious sects, convents and other organizations?

Systems of values can be studied starting with general meaning determining what is true, good and nice versus what is false, bad and ugly. The concept of general meaning would be less significant if it did not have anything to say about conversion of general meaning into detailed meaning and pragmatics. However, before we consider this issue I want to turn into the next step of our concept. Having established the first step i.e. identifying a scale or scales which is proper for a given utterance we want to resort to the placement of the utterance on a scale or scales. Location on scale or scales is an important part of semantic description of an utterance.

We do not have a hard and fast way of placing an utterance on a definite place on the scale or scales. It would rather be impossible to find such principle and moreover it would not reflect perceptual nature of the phenomenon. Assessment on the scale is necessarily fuzzy, since individual and contextual factors are indeterminate in numerical or digital fashion. However, what is possible is presentation of tendencies of alignment of a given utterance at a given place on the scale. A proposal for checking location on a scale is connected with a parenthetical test. Negative and positive parentheticals are added to a given utterance. The results can show tendencies concerning location on a given scale or scales. The area on the spectrum can be tentatively established. The examples of parentheticals for three scales include: but it may be the case, but it may not be the case, and it may be the true, and it may not be true for truth-falsehood scale; but I like it, but I don't like it, and I like it, and I don't like it, and it is good, and it isn't good, but it is good, it isn't good for positive-negative scale: finally and it is beautiful, and it is not beautiful, but it is ugly, but it is not ugly for the beautiful-ugly scale.

The basic results of adding parentheticals like the above show when we have to do with utterances located near the poles of a scale. A positive parenthetical causes tautology with utterances close to a positive pole of a scale and a negative parenthetical causes contradiction with such utterances.
a. *I'm sure that Mary left, but it may be true - taut ology
b. *I'm sure that Mary left, but it may not be true - contradiction
a. *It is impossible that Mary left, but it may not be true - tautology
b. *It is impossible that Mary left, but it may be true - contradiction

In the second couple of utterances the situation is reversed i.e. a negative parenthetical leads to tautology and positive contradiction. This behavior shows that we have to do with an utterance representing strong conviction of the author of the message concerning falsehood of the proposition i.e. close to the negative pole of the scale. In utterances which are closer to the middle of the scale, the situation is different:

a. *I think that Mary left, but it may be the case - tautology

b. I think that Mary left, but it may not be the case - O.K. 
In the first case of the above couple a positive parenthetical causes tautology showing that an utterance should be located on the positive end of the scale. However, the second case, where the sentence is grammatical with negative parenthetical, shows that the utterance should rather be located on the positive half of the scale. However, the second case, where the sentence is grammatical with negative parenthetical, shows that the utterances should rather be located closer to the middle of the scale than closer to the positive pole, since the conviction concerning the truth of the proposition is not very strong, allowing challenging of the statement. We have a reversed situation in the cases below:

$$
\begin{aligned}
& \text { a. I doubt whether Mary left, but it may be the case - O.K. } \\
& \text { b. *I doubt whether Mary left, but it may not be the case - tautology }
\end{aligned}
$$

The utterance with doubt should be located on the negative half of the scale, however, closer to the middle than to the negative pole, since positive parenthetical does not yield ungrammaticality.

A similar situation is met in other scales:

$$
\begin{aligned}
& \text { a. *I'm happy that I work in Gda ńsk, but I like it - tautology } \\
& \text { b. *I am happy that I work in Gdańsk, but I don't like it - contradiction }
\end{aligned}
$$

There is also a different relation of an utterance toward placement on the positive negative scale:
a. It is bad that you failed your exam
b. It's not that awful that you failed your exam

Having determined a proper scale or scales for a given utterance and locating it on a certain area of such scale or scales, it is time to show how such general meaning is converted into concrete lexical meaning. The directionality of analysis, i.e. from general to detailed meaning, does not seem to be important here; however, in this paper we will employ a deductive method. In other words, we will start with general meaning and we will translate it into or instantiate it as a detailed lexical meaning. Summing up what we have maintained so far: we identify a scale or scales, present a tentative placement of the expression on one or more scales and convert general meaning already partly determined into a detailed meaning at best to the level of a morpheme. It is the starting stage of this account, so the analysis is neither conclusive nor complete. It only presents a suggestion toward a different way of semantic analysis. The essence of the analysis is finding an exponent or exponents of particular scale or scales and building a semantic skeleton for the utterance involving construction meaning (cf. Goldberg 1995), lexical and morphological meaning. A type of sentence construction contributes to a sentence meaning. 
A simple sentence consisting of one clause does not constitute a great problem for our analysis. Such a sentence immediately imposes a very high conviction of the author of the utterance concerning the truth of the analyzed sentence. For instance,

The train arrived at six

The above sentence shows very high conviction that an object (here train) came to a given location at some time (here six). Nevertheless, although a simple sentence without modification triggers inevitably truth - falsehood scale where the truth of the proposition is highly stressed, still other scales are not blocked e.g.

Christine loves sushi.

In the above sentence the truth of Christine's love for sushi is highly claimed, but it does not exhaust perceptual scales of general meaning. Love is a strong indication for semantic skeleton to the effect that Christine has a very strong, positive feeling toward eating a famous Japanese dish.

This is a very beautiful Monet.

The above sentence also contains an aesthetic scale. Thus it can be seen from the above examples that a simple sentence consisting of one clause imposes truth - falsehood scale pointing at a very high deg ree of conviction concerning the truth of the proposition contained in the clause, but it does not block other scales and admits variety of assessment on other scales, e.g.

This soup is awful.

Other syntactic constructions bring in their construction meaning connected with triggering of scales and placement of the utterances on particular areas of the scales.

Simple sentences with modal expressions as the term itself suggests, modify a statement contained in the expression in terms of truth - falsehood, positive - negative, beauty - ugliness. In the scal e expressing truth - falsehood it often weakens the strength of conviction ( however it does not always have to be so since we have such expressions as certainly, surely, etc.).

(14) Maybe the train has arrived.

It's likely that Mary bought this dress.

Other modal expressions such as Polish wspaniale, świetnie, cudownie or English wonderfully, greatly, it's beautiful which are connected with other scales are separated from the simple sentence in an iconic manner by intervention of conjunction that in English or $\dot{z} e$ in Polish between a modal expression (most often an adverb or a modal phrase containing $b e$ ) and the clause without modification. 
(16) Wspaniale, że ugotowałaś obiad.

'Wonderfully that you cooked-fem dinner.'

It is wonderful that you cooked dinner.

More complex constructions are also susceptible to the above analysis. See below a sentence with adverbial clauses:

If I got well, I would visit you.

The analysis is as follows: If a given condition is fulfilled, i.e. if something good happened - if the speaker got well, then something would happen in the world - the speaker would visit the addressee. Here we have to do with two scales i.e. truth falsehood and positive - negative.

The inventories of constructions presented in various grammars are analyzable according with the above directions.

\section{The concept of general meaning with respect to selected linguistic theories}

\subsection{General meaning and axiological semantics}

It may be claimed that our concept of general meaning is a variant of axiological semantics. This may be partly true because we deal with values and their role in language. Nevertheless, the role and the number of values in the concept of general meaning differs significantly from Krzeszowski's (1997) and Puzynina's (1992) account.

Krzeszowski (1997) maintains that there are two basic values, i.e. good and bad, and that every expression in every language is axiologically charged,namely having good bad values. I agree with Krzeszowski however only in the contention that any expression in every language may be axiologically loaded (in Krzeszowski's (1997) account bearing good or bad value), since it is always possible to provide a context that would trigger good - bad evaluation. Thus good leaf may be a mysterious construction, however, in a cigar factory, it is an obvious and natural expression.

It would be difficult to give a good - bad interpre tation to such expressionsas $A$ triangle has three angles or Venus is the second plannet of the solar system without special contextual conditions. Hence, we contend that it is not always that good - bad relation is present in i nterpretation of utterances. However, it is the case that every linguistic expression possesses general meaning connected with true - false, positive negative, beautiful - ugly parameters.

It may be argued that some scales may be reduced to a good - bad scale. It is possible for the scale beautiful - ugly where a beautiful object is positive and ugly is purportedly negative. However, there is nothing inherently good in Mary in the utterace like Mary is beautiful and cruel or bad in ugliness: Poor, ugly Jane has a good heart. 
There is nothing good in Oil flows on water, although the sentence is true. Obviously it is possible to find context where flowing of oil on water is beneficial but it is not possible to claim that truth is always reducible to good or false to bad. Therefore we claim that account based on general meaning is more encompassing than Krzeszowski's (1997) axiological principle.

\subsection{Wierzbicka's atomic expressions and the concept of general meaning}

In her monumental work Wierzbicka $(1969,1980,1987$ and many, many other) develops Leibnitz's idea of atomic expressions which are also called indefinibilia, alphabet of human thought, primes and universals etc. The idea Wierzbicka entertains is that any expression in any language can be translated into a language consisting exclusively of terms which are not analyzable any further. This enterprise is not possible (see Kalisz 2001) with thirty two atomic expressions. The analyses conducted by Wierzbicka almost always involve intermediate expressions taking for granted that they can be split into atomic expressions with ease. This is not true. Attempts to get to ultimate atomic expressions almost in every case are doomed to failure because of such phenomena as Gestalt, impossibility to finish explication, awkwardness or unreadibility. The present author has not examined most recent Wierzbicka's inventory which at present consists of more than sixty atomic expressions (the smallest number was thirteen in Wierzbicka 1980).

Leaving the plausibility of Wierzbicka's analyses aside we may contend that her attempt is directed toward lower semantic units than lexical items. In other words, it is going beyond the meaning a of lexical item. Our account is headed in the opposite direction. We try to determine a general meaning above a detailed meaning of a lexical item. Our attempt is not contradictory with respect to Wierzbicka's research. An analysis of a lexical item, phrase or a sentence may start with general meaning reaching lexical meaning level ending with atomic expressions (or the other way around). This is theoretically possible and may be fruitful for a profound semantic analysis of a linguistic expression.

\subsection{General meaning and meaning in classical pragmatics}

\subsubsection{Speech act theory and the concept of general meaning}

Linguistic pragmatics deals with general meaning but in a slightly different sense than CGM (concept of general meaning). In a pragmatic concept of language we analyze types of speech acts (speech act theory), indirect meaning (theory of conversational implicature or theory of indirect speech acts) which are not exclusively determined by lexical meanings of words in an utterance. It is written here 'exclusively' because some semantic relation has to be present between lexical meaning and indirect speech act or a type of a speech act. 
The following expressions may serve the speaker to make the addressee close the window in the room:
a. Could you close the window?
b. It's cold in here!
c. Syberia!
d. My radicles!

However the utterances below cannot serve such purpose, unless they constitute a special code between the speaker and the addressee:
a. Tomorrow I'm going to watch a controversial movie.
b. Cristine really likes Peter

It is difficult (in fact impossible) to derive meaning connected with closing the window since lexical items and in fact their meanings do not lead to understanding of discomfort of the author of the statement connected with cold arising from air flow through the open window.

However in pragmatics such as in CGM we note both general meaning (promise, reprimand, request for closing the window) and detailed meaning. Do you have to make such noise represents a reprimand or It's cold in here which represents a pragmatic request for closing the window. The existing semantic relation between lexical meaning and general pragmatic meaning is important here. The attempts of analysis of such relations were conducted although from a different perspective. For example, Verschueren (1977) presents what he calls speech act verbs. He wants to unify acts with existing verbs in English e.g. V - act of V - ing. i.e. act of doubt - ing for the verb doubt or act of judging for the verb judge. The problems here are created by indirect speech acts and expressions which are close in meaning. E.g. act of claiming (that p) and stating (that $\mathrm{p}$ ). It is an attempt to connect general pragmatic meaning with lexical meaning with lexical meanings $i$. e. verb meaning = meaning type of a speech act. The Verschueren's ideas to differentiate the largest number of speech acts reminding astronomical number of spiders and beetles distinguished by biologists.

In our account we draw consciously a border between a general meaning of an utterance and detailed lexical meaning contending that general meaning can be transferred into detailed lexical meaning. The transfer works in both directions i. e it is possible to generalize a detailed meaning as in I greeted her (saying 'Good morning ' to her ) or Good morning Christine represents a detailed form of greeting. In our analysis we start from general meaning and by creation of semantic skeleton we arrive at detailed lexical meaning. Wierzbicka (e.g. 1969, 1980, 1987 and others) takes a reversed road.

Searle $(1969,1977)$ classification of speech acts and classifications of other scholars show series of verbal behaviors of people. Although Wierzbicka (1987) characterizes speech act theory as anglocentric, still in the majority of languages one can distinguish promises or directives as identified by Searle (1969, 1977). They constitute general 
meanings of utterances. Obviously, it is possible to distinguish various levels of pragmatics as attempted in Verschueren (1977) and in his postulate to distinguish many speech acts e.g. promise and unsolicited promise (see Kalisz 1993). Those meanings are not identical with general meanings postulated in CGM. However, they have many properties in common. A similar phenomenon is a level of abstraction of a speech acts and spectrum positive - negative or true - false. Searle's (1977) assertion is connected with conviction of the speaker concerning the truth contained in the proposition (in varying degrees). Reprimand or accusation would represent positive - negative scale, close to the negative pole, whereas congratulations would have a different orientation on this scale i. e. drifting toward positive pole. Expressives such as What a wonderful view represent the beautiful - ugly scale.

Thus, it can be seen that relation between a speech act type and CGM scale(s) is possible to establish. They represent a similar level of generality in analysis of meaning. Pragmatic meaning of an utterance is its general meaning representing a certain type of a speech act.

\subsubsection{CGM and theory of conversational implicature}

Conversational implicature theory (Grice 1975 and others) is aimed at discovery of meaning often hidden behind lexical meanings of utterances. Original Grice's example involves one professor asking another professor who had been Jones's philosophical teacher about Jones' s philosophical knowledge. The teacher's answer Grice's conversational maxims stating that Jones's handwriting was excellent and his attendance to tutorials had been regular. Jones's teacher in spite of that he breaks maxims (here maxims of Quantity and Relation), still preserves the cooperative principle. At least the professor who is to employ Jones understands it the way which allows him to derive implicature 'Jones is not good in philosophy'. This message suffices the professor who is to employ Jones at least for some time which is decisive for Jones's fate.

It has to be noted that the calculated meaning is very close to general meaning concerning the conviction toward the truth of the proposition and its negative impact.

Another Gricean example may be provided: How is Peter? Oh, he likes his colleagues and he hasn't been to prison yet! Conversational implicature is as follows: 'Peter is in a dangerous company'.

Not only the positive - negative spectrum is evoked by conversational implicature, e.g. Andrew and Adalbert are excellent friends used in the situation where both men behaved awfully with respect to the speaker. This case represents irony which is representative of the truth - falsehood scale, obviously apart from shameful behavior of Andrew and Adalbert. Conversational implicature triggers the scales which are provided by CGM.

\subsubsection{Theory of relevance and CGM}

Sperber and Wilson (1986, 1995 and others ) select one of Gricean ( 1975 ) maxims of conversation, namely relevance ( originally maxim of relation ) and build around it the 
whole theory of pragmatics which is later translated into truth conditional semantics and its anchoring in context. An assumption is more relevant if its contribution to conversation is very significant and its understanding/processing does not require much effort. Therefore, with high values of both parameters the assumption is highly relevant and of little relevance when the two values are low. The aim of pragmatics is a description of the speaker's meaning and filling the gap between linguistic meaning and intentions which are hidden behind this meaning (Mioduszewska 2006: 169 ).

Pragmatics of relevance operates with explicature and implicature. The first conclusions stem from lexical meanings of utterances and implicature, as in Grice, deals with indirect meaning. Thus, implicature is an ultimate goal searched by a language user. Explicature is the means of understanding implicature, in spite of that it has the meaning of its own in looking for relevance. It is possible here to equate general meaning with implicature where explicature may be treated as exposition of detailed lexical meaning.

Analyzing expressions such as:

a. Shall we go to the movies tonight ?

b. I have an exam tomorrow!

it is possible to show the way of A's reasoning leading to the discovery of B's intention. On the basis of explicature, A expects an answer to his/her question from B. B says that he/she has an exam the next day. A's reasoning is roughly as follows: studying for an exam takes a lot of time, therefore it is not possible for B to do both i.e. to go to the movies and to study for the exam. Hence, B's intention is refusal to go to the movies. The reasoning of $\mathrm{A}$ is so far very similar to calculation of conversational implicature. The difference here is the notion of explicature which denotes parallel reasoning with derivation of implicature. Explicature may contain larger context connected with honesty of B i.e. whether B really has an exam the next day or B simply does not want to go to the movies with that person and the exam is only a purported argument. As in the case of conversational implicature, an indirect meaning is more general than lexical meaning

\section{Epilogue}

The above section deals with comparison of concept of general meaning (CGM) with linguistic theories which are closest to our account. We selected two semantic and three pragmatic theories. The pragmatic theories considered here are classical i.e. speech act theory, conversational implicature and relevance theory. We chose classical theories in their form because recent pragmatic research is more connected with sociolinguistic phenomena and other fields like discourse or specialized language. Even Verschueren (2003), formerly a noted speech act theoretician writes recently papers on such topics as erasion of group - based social inequality. We want to go to the roots of the above pragmatic theories because it is more useful in comparing the accounts. 


\section{References}

Brown Penelope and Stephen C. Levinson. 1997. Politeness: some universals in language use. Cambridge: Cambridge University Press.

Grice, H.P. 1975. 'Logic and conversation' In: Cole . P. and J. L. Morgan. (ed.). Syntax and semantics 3: speech acts. New York: Academic Press, $41-58$.

Kalisz, Roman. 1993. Pragmatyka językowa. Gdańsk: Wydawnictwo U.G.

Krzeszowski, Tomasz P. 1997. Angels and Devils in Hell: Elements of Axiology in Semantics. Warszawa: Energeia.

Mioduszewska, Ewa. 2006. 'Teoria relewancji'. In: Stalmaszczyk, P. (ed.). Metodologie językoznawstwa. Podstawy teoretyczne. Łódź: Wydawnictwo U.Ł., 155 - 173.

Puzynina, Jadwiga. 1992. Język wartości. Warszawa: PWN.

Searle, John R. 1969. Speech acts. Cambridge : CUP.

Searle, John R. 1977. 'A classification of illocutionary acts" In: Rogers,A, et al. (eds.). Proceeding of the Texas conference on performatives, presuppositions and implicatures. Arlington, Va.: Center for Applied Linguistics.

Verschueren, Jef. 1977. 'Speech act verbs”. Indiana U niversity Linguistic Club.

Verschueren Jef. 2003. 'A touch of class: the erasion of group - based social inequality as a homogenuous process in political discourse'.

Pragmatics, vol 13 no.1, $135-134$.

Wierzbicka, Anna. 1969. Dociekania semantyczne. Wrocław: Ossolineum.

Wierzbicka, Anna. 1980. Lingua mentalis. New York: Academic Press.

Wierzbicka, Anna. 1987. Lexicography and conceptual analysis. Ann Arbor: Karoma Publishers. 